EINDHOVEN UNIVERSITY OF TECHNOLOGY

Department of Mathematics and Computing Science

\author{
RANA $92-03$ \\ March 1992 \\ ON MAXIMUM NORM CONVERGENCE OF \\ MULTIGRID METHODS FOR ELLIPTIC \\ BOUNDARY VALUE PROBLEMS \\ by
}

A. Reusken 
Reports on Applied and Numerical Analysis

Department of Mathematics and Computing Science

Eindhoven University of Technology

P.O. Box 513

$5600 \mathrm{MB}$ Eindhoven

The Netherlands

ISSN: 0926-4507 


\title{
On Maximum Norm Convergence of Multigrid Methods for Elliptic Boundary Value Problems
}

by

\author{
Arnold Reusken
}

\begin{abstract}
We consider multigrid methods applied to standard linear finite element discretizations of linear elliptic boundary value problems in $2 D$. In the multigrid method damped Jacobi or damped Gauss-Seidel is used as a smoother. We prove that the two-grid method with $\nu$ pre-smoothing iterations has a contraction number with respect to the maximum norm that is (asymptotically) bounded by $C \nu^{-\frac{1}{2}}\left|\ln h_{k}\right|^{2}$, with $h_{k}$ a suitable mesh size parameter. Moreover, it is shown that this bound is sharp in the sense that a factor $\left|\ln h_{k}\right|$ is necessary.
\end{abstract}

Key words. Multigrid, convergence analysis, maximum norm, elliptic boundary value problems.

AMS (MOS) subject classification. 65 N20 


\section{Introduction}

If one considers elliptic boundary value problems in $\mathbb{R}^{N}(N=2,3)$ then multigrid methods can be used to solve efficiently the large sparse linear systems that arise after discretization. In recent years there has been intensive research into the theoretical understanding of the convergence properties of these methods. We refer to Hackbusch [10], McCormick [14] and the references therein. The main feature of multigrid is that for a broad class of problems the contraction number has an upper bound which is smaller than one and independent of the mesh size. In theoretical analyses this has been shown for several variants of multigrid. Usually in these analyses the energy norm is used; sometimes one uses the euclidean norm. Some first results about multigrid convergence in the maximum norm are presented in [19]. In that paper, however, only two-point boundary value problems are treated. In this paper we present convergence results in the maximum norm for multigrid applied to a class of elliptic two dimensional boundary value problems. We consider a regular linear (nearly) symmetric elliptic boundary value problem on a domain $\Omega \subset \mathbb{R}^{2}$ and we use linear finite elements on quasi-uniform triangulations.

Two main results of this paper are the following. Firstly, we prove that for a two-grid method with $\nu$ damped Jacobi or damped Gauss-Seidel smoothing iterations the contraction number with respect to the maximum norm is (asymptotically) bounded by $C \nu^{-\frac{1}{2}}\left|\ln h_{k}\right|^{2}$ (with $h_{k}$ a suitable mesh size parameter). Secondly, it is shown that this bound is sharp in the sense that a factor $\left|\ln h_{k}\right|$ is necessary: For a concrete (very regular) example we prove that the contraction number with respect to the maximum norm of a standard two-grid method with a fixed number of smoothing iterations is bounded from below by $C\left|\ln h_{k}\right|$.

So instead of an "optimal" bound $C \nu^{-1}$ for the contraction number in the energy norm (or the euclidean norm) we obtain a "nearly optimal" bound $C \nu^{-\frac{1}{2}}\left|\ln h_{k}\right|^{2}$ if we use the maximum norm and this bound is sharp in some way.

We now outline the remainder of this paper. In $\$ 2$ we introduce a class of elliptic boundary value problems. Some properties of the usual linear finite element discretization on a sequence of quasi-uniform triangulations are derived in $\S 3$. An important property, due to Descloux [7], is that the mass matrix has a condition number which is uniformly bounded (for $h_{k} \downarrow 0$ ) with respect to the maximum norm. In $\S 4$ and $\S 5$ we prove the Approximation Property and Smoothing Property (cf. Hackbusch [10]) respectively. In the proof of the Approximation Property we use a regularity result due to Campanato [5] and an $L^{\infty}$-finite element error estimate due to Rannacher and Frehse $[18,8]$. From the latter the factor $\left|\ln h_{k}\right|^{2}$ originates. The Smoothing Property is proved using a new technique introduced in [20]. In $\S 6$ we derive convergence results for the two-grid method and we discuss the multigrid $W$-cycle. Finally, in $\S 7$, we analyze a specific example. We consider the Poisson equation on the unit square and use a linear finite element discretization on a uniform triangulation. We prove a $C\left|\ln h_{k}\right|$ lower bound for the contraction number of a standard two-grid method with a fixed number of smoothing iterations. Our analysis in $\S 7$ is based on the approach used by Haverkamp in [11].

\section{Continuous problem}

Let $\Omega \subset \mathbb{R}^{2}$ be a bounded open domain with $\partial \Omega$ sufficiently smooth. We consider the following variational boundary value problem: 


$$
\left\{\begin{array}{l}
\text { find } \varphi^{*} \in H_{0}^{1}(\Omega) \text { such that for all } \psi \in H_{0}^{1}(\Omega) \\
\sum_{i, j=1}^{2} \int_{\Omega} a_{i j} \frac{\partial \varphi^{*}}{\partial x_{i}} \frac{\partial \psi}{\partial x_{j}} d x=\int_{\Omega} f \psi d x
\end{array}\right.
$$

We use the notation

$$
a(\varphi, \psi)=\sum_{i, j=1}^{2} \int_{\Omega} a_{i j} \frac{\partial \varphi}{\partial x_{i}} \frac{\partial \psi}{\partial x_{j}} d x \quad\left(\varphi, \psi \in H_{0}^{1}(\Omega)\right),
$$

and we make the following assumptions about $\Omega, f, a(\cdot, \cdot)$ :

(2.4.a) $\left.\left.\quad \partial \Omega \in C^{2, \alpha} \quad(\alpha \in] 0,1\right]\right)$

(2.4.b) $\left.\left.\quad a_{i j}=a_{j i} ; a_{i j} \in C^{1, \alpha}(\bar{\Omega}) \quad(\alpha \in] 0,1\right]\right)$

(2.4.c) there are constants $\lambda_{1}, \lambda_{2}>0$ such that for all $\left(\xi_{1}, \xi_{2}\right) \in \mathbb{R}^{2}$ and all $x \in \Omega$

$\lambda_{2}|\xi|^{2} \leq \sum_{i, j=1}^{2} a_{i j}(x) \xi_{i} \xi_{j} \leq \lambda_{1}|\xi|^{2}$.

By $H^{m, p}(\Omega), H_{0}^{m, p}(\Omega)(1 \leq p \leq \infty, m \in \mathbb{N})$ we denote the usual Sobolev spaces with norm $\|\cdot\|_{H^{m, p}}$ and $\|\cdot\|_{H_{0}^{m, p}}$ respectively. If $p=2$ we use the notation $H^{m}(\Omega), H_{0}^{m}(\Omega)$.

Remark 2.1. In multigrid convergence theory an important role is played by the regularity of the differential operator. It is well-known that under the assumptions (2.3), (2.4.a-c) we have $H^{2}$-regularity: $\varphi^{*}$ is an element of $H^{2}(\Omega) \cap H_{0}^{1}(\Omega)$ and $\left\|\varphi^{*}\right\|_{H^{2}} \leq c\|f\|_{L^{2}}$ with $c$ independent of $f$. If instead of $p=2$ we take another $p \in] 1, \infty\left[\right.$ then a similar $H^{2, p}$-regularity result holds (cf. [9]). However, even if $\partial \Omega$ and the coefficients $a_{i j}$ are very smooth, a similar $H^{2, \infty}$-regularity estimate $\left\|\varphi^{*}\right\|_{H^{2, \infty}} \leq c\|f\|_{L^{\infty}}$ does not hold, as is shown by the following example (due to [17]). Let $\Omega$ be the unit sphere and $a(\varphi, \psi):=\int_{\Omega} \nabla \varphi \cdot \nabla \psi d x$. For $\varepsilon \in[0,1]$ we define $\varphi_{\varepsilon}^{*}\left(x_{1}, x_{2}\right)=x_{1} x_{2} \ln (|x|+\varepsilon)-x_{1} x_{2} \ln (1+\varepsilon)$ (with $\left.\varphi_{0}^{*}(0,0):=0\right)$. Then $\varphi_{\varepsilon \mid \partial \Omega}^{*}=0$ and $\Delta \varphi_{\varepsilon}^{*}$ exists on $\Omega$. Now define $f_{\varepsilon}:=-\Delta \varphi_{\varepsilon}^{*}$, then $f_{\varepsilon} \in L^{\infty}(\bar{\Omega})$ for all $\varepsilon \in[0,1]$ and even $f_{\varepsilon} \in C(\bar{\Omega})$ if $\left.\left.\varepsilon \in\right] 0,1\right]$. However, if $\varepsilon=0$ we have $\varphi_{\varepsilon}^{*} \notin H^{2, \infty}(\Omega)$ and if $\left.\left.\varepsilon \in\right] 0,1\right]$ we have $\varphi_{\varepsilon}^{*} \in H^{2, \infty}$ but $\left\|\varphi_{\varepsilon}^{*}\right\|_{H^{2, \infty}}\left\|f_{\varepsilon}\right\|_{L^{\infty}}^{-1}$ is unbounded for $\varepsilon \downarrow 0$.

In the proof of the Approximation Property $(\S 4)$ w.r.t. the $L^{\infty}$-norm another type of regularity result (due to Campanato [5]) is used, in which the usual $L^{p}(\Omega)$ space is replaced by the space of John-Nirenberg $E^{0}(\Omega)$ (also called the space of functions of bounded mean oscillation, cf. [12]). This space satisfies $L^{\infty}(\Omega) \stackrel{\ulcorner}{\neq} E^{0}(\Omega) \stackrel{\complement}{\neq} L^{p}(\Omega)$ for all $p \in[1, \infty[$.

\section{Discretization and two-grid method}

We take a sequence of nested quasi-uniform triangulations $\left\{\mathcal{T}_{k} \mid k \in \mathbb{N}_{0}\right\}$ as follows. For every $k$ we define $\Omega_{k}:=\cup_{T \in \mathcal{T}_{k}} T$ and for every $\mathcal{T}_{k}$ we use a mesh size parameter $h_{k}$ with $0<h_{k+1}<h_{k}<1$. We make the following assumptions (with constants $c_{i}$ independent of $T$ and $k$ ): 
(3.1.a) $\operatorname{dist}\left(\partial \Omega_{k}, \partial \Omega\right) \leq c_{0} h_{k}^{2}$

(3.1.b) for any two different triangles in $\mathcal{T}_{k}$ the intersection is empty or consists of a common vertex or of a common side;

(3.1.c) for every $T \in \mathcal{T}_{k}$ there is a disc with radius $c_{1} h_{k}$ containing $T$ and a disc with radius $c_{2} h_{k}$ contained in $T$;

(3.1.d) for every $\hat{T} \in \mathcal{T}_{k}$ there is a subset $S$ of $\mathcal{T}_{k+1}$ such that $\cup_{T \in S} T=\hat{T}$ (this corresponds to mesh refinement);

(3.1.e) $\quad h_{k} h_{k+1}^{-1} \leq c_{3}$.

Continuous piecewise linear functions on such a triangulation $\mathcal{T}_{k}$ yield a finite dimensional function space

$$
\Phi_{k}=\left\{v \in C(\bar{\Omega}) \mid v \text { is linear on every } T \in \mathcal{T}_{k} \text { and } v \equiv 0 \text { on } \partial \Omega_{k} \cup\left(\bar{\Omega} \backslash \Omega_{k}\right)\right\}
$$

These spaces are nested, i.e.

$$
\Phi_{0} \subset \Phi_{1} \subset \ldots \subset \Phi_{k} \subset \ldots \subset H_{0}^{1}(\Omega)
$$

The collection of interior grid points in $\mathcal{T}_{k}$ is denoted by $\left\{x_{k}^{i}\right\}_{i \in J_{k}}$ for some indexset $J_{k}$ with $\# J_{k}=n_{k}$. We use the notation $U_{k}=\mathbb{R}^{n_{k}}$. The standard basis of $\Phi_{k}$ is given by the functions $\varphi_{k}^{i} \in \Phi_{k}$ which satisfy $\varphi_{k}^{i}\left(x_{k}^{j}\right)=\delta_{i j}\left(i, j \in J_{k}\right)$. This induces the natural bijection

$$
P_{k}: U_{k} \rightarrow \Phi_{k}, \quad P_{k}(u)=\sum_{i \in J_{k}} u_{i} \varphi_{k}^{i}
$$

On $U_{k}$ we use a scaled euclidean inner product with corresponding norm

$$
<u, v>_{k}=h_{k}^{2} \sum_{i \in J_{k}} u_{i} v_{i},\|u\|_{k}=<u, u>_{k}^{\frac{1}{2}}
$$

The maximum norm on $U_{k}$ is denoted by $\|\cdot\|_{\infty}$. Below, adjoints are always defined with respect to the $L^{2}$-inner product on $\Phi_{k}$ and $\langle\cdot, \cdot\rangle_{k}$ on $U_{k}$. The norms $\|\cdot\|_{\infty}$ (on $U_{k}$ ) and $\|\cdot\|_{L^{\infty}}\left(\right.$ on $\Phi_{k}$ ) induce associated operator norms which are denoted by $\|\cdot\|_{\infty}$.

Remark 3.1. Lemma 3.2 below yields that the sequences $\left(P_{k}\right)_{k \geq 0},\left(P_{k}^{-1}\right)_{k \geq 0},\left(P_{k}^{*}\right)_{k \geq 0}$ and $\left(\left(P_{k}^{*}\right)^{-1}\right)_{k \geq 0}$ are uniformly bounded in $\|\cdot\|_{\infty}$. In the analysis of multigrid convergence in the euclidean norm (cf. [10]) such a uniform boundedness result is used too, but then w.r.t. the norms $\|\cdot\|_{k}$ and $\|\cdot\|_{L^{2}}$. The latter uniform boundedness is closely related to the well-known fact that the mass matrix $P_{k}^{*} P_{k}$ has a condition number $O(1)\left(h_{k} \downarrow 0\right)$ in the euclidean norm. The main argument in the proof of Lemma 3.2 is that the condition number of the mass matrix is $O(1)\left(h_{k} \downarrow 0\right)$ w.r.t. the maximum norm, too. This result has been proved by Descloux in [7].

Lemma 3.2. Assume (3.1.b,c), then the following holds with constants $C_{1}$ and $C_{2}$ independent of $k$ : 
(1) $\quad\left\|P_{k} u\right\|_{L^{\infty}}=\|u\|_{\infty}$ for all $u \in U_{k}$,

(2) $\quad C_{1}\|\varphi\|_{L^{\infty}} \leq\left\|P_{k}^{*} \varphi\right\|_{\infty} \leq C_{2}\|\varphi\|_{L^{\infty}}$ for all $\varphi \in \Phi_{k}$.

Proof. The result in (1) holds because $P_{k} u$ is piecewise linear and $\left(P_{k} u\right)\left(x_{k}^{i}\right)$ equals the $i$-th component of $u$. Due to (1) the statement in (2) is equivalent with

$$
C_{1}\|u\|_{\infty} \leq\left\|P_{k}^{*} P_{k} u\right\|_{\infty} \leq C_{2}\|u\|_{\infty} \text { for all } u \in U_{k}
$$

For $i, j \in J_{k}$ we have (with $e_{m}$ the $m$-th unit vector in $U_{k}$ )

$$
\left(P_{k}^{*} P_{k}\right)_{i, j}=h_{k}^{-2}<P_{k}^{*} P_{k} e_{j}, e_{i}>_{k}=h_{k}^{-2} \int_{\Omega} \varphi_{k}^{i} \varphi_{k}^{j} d x
$$

So, using (3.1.c), we get

$$
\left\|P_{k}^{*} P_{k}\right\|_{\infty}=\max _{i} h_{k}^{-2} \sum_{j \in J_{k}} \int_{\Omega} \varphi_{k}^{j} \varphi_{k}^{i} \leq \max _{i} h_{k}^{-2} \operatorname{supp}\left(\varphi_{k}^{i}\right) \leq C_{2},
$$

thus the second inequality in (2') holds. For the first inequality in (2') we note that due to the quasi-uniformity of the triangulations the assumptions in [7] hold. Theorem 2 in [7] then yields the desired result.

Galerkin discretization results in a stiffness matrix $L_{k}: U_{k} \rightarrow U_{k}$ defined by

$$
<L_{k} u, v>_{k}=a\left(P_{k} u, P_{k} v\right) \text { for all } u, v \in U_{k} \text {. }
$$

Also we have that

$$
a\left(P_{k} L_{k}^{-1} g, P_{k} v\right)=\left\langle g, v>_{k} \text { for all } g, v \in U_{k}\right.
$$

For solving a system of the form $L_{k} u_{k}=g_{k}$ we use a standard multigrid method. The iteration matrix of the smoothing method is denoted by $S_{k}$. For the prolongation $p=p_{k}: U_{k-1} \rightarrow U_{k}$ we use the natural one:

$$
p=P_{k}^{-1} P_{k-1}
$$

For the restriction $r=r_{k}: U_{k} \rightarrow U_{k-1}$ we take

$$
r=p^{*}
$$

The iteration matrix of the two-grid method with $\nu$ pre-smoothing iterations is given by

$$
T_{k}(\nu)=\left(I-p L_{k-1}^{-1} r L_{k}\right) S_{k}^{\nu}=\left(L_{k}^{-1}-p L_{k-1}^{-1} r\right) L_{k} S_{k}^{\nu}
$$


Below, in $\S 4$ and $\S 5$ we will prove the Approximation Property and Smoothing Property (cf. [10]) with respect to the norm $\|\cdot\|_{\infty}$.

\section{The Approximation Property}

We begin this section with a discussion of a rather special finite element $L^{\infty}$-error estimate that will be used in the proof of the Approximation Property below.

In most convergence analyses of multigrid the regularity of the underlying boundary value problem is used. If the analysis is based on the energy norm then $h$-independent convergence can be proved under very weak regularity conditions (cf. eg. [1], [2], [3], [4], [10], [13], [16]). If, however, one wants to prove the Approximation Property in the scaled euclidean norm then an $H^{2}$-regularity estimate

$$
\left\|\varphi^{*}\right\|_{H^{2}} \leq c\|f\|_{L^{2}}
$$

is necessary, as is shown in [6]. The Approximation Property then follows from a combination of (4.1) with the following (in which $\varphi_{k}^{*}$ is the Galerkin solution in $\Phi_{k}$ ):

$$
\begin{aligned}
& \text { (4.2.a) }\left\|\varphi_{k}^{*}-\varphi^{*}\right\|_{L^{2}} \leq c h_{k}^{2}\left\|\varphi^{*}\right\|_{H^{2}} \text { (finite element error estimate), } \\
& \text { (4.2.b) }\|\cdot\|_{L^{2}} \text { and }\|\cdot\|_{k} \text { are uniformly equivalent for } k \rightarrow \infty
\end{aligned}
$$

In this paper we want to prove the Approximation Property in the maximum norm. Clearly, the analogue of (4.2.b) is given in Lemma 3.2. With respect to (4.2.a) we note that $L^{\infty}$-error estimates of the form

$$
\left\|\varphi_{k}^{*}-\varphi^{*}\right\|_{L^{\infty}} \leq c h_{k}^{2}\left|\ln h_{k}\right|\left\|\varphi^{*}\right\|_{H^{2, \infty}}
$$

can be found in the literature (e.g. in [8], [15], [22], [23]). However, it is shown in Remark 2.1 that, even for very regular problems, an $H^{2, \infty}$-regularity estimate $\left\|\varphi^{*}\right\|_{H^{2, \infty}} \leq c\|f\|_{L^{\infty}}$ does not hold. Therefore it is not clear how (4.3) can be used to prove the Approximation Property. In $[8,18]$ Rannacher and Frehse prove the following type of (asymptotic) error estimate for $f \in L^{\infty}$ :

$$
\left\|\varphi_{k}^{*}-\varphi^{*}\right\|_{L^{\infty}} \leq c h_{k}^{2}\left|\ln h_{k}\right|^{2}\|f\|_{L^{\infty}}
$$

This result, which is a substitute for the combination of (4.1) and (4.2.a), and the result of Lemma 3.2 are the main points in the proof of the Approximation Property in Theorem 4.2 below.

Remark 4.1. We briefly comment on the combination of regularity and approximation properties of $\Phi_{k}$ used in the proof of (4.4). Instead of $L^{p}(\Omega)$ the John-Nirenberg space $E^{0}(\Omega)$ is used with a suitable norm denoted by $\|\cdot\|_{L^{2,0}}$. For $E^{0}(\Omega)$ one has $L^{\infty}(\Omega) \stackrel{c}{\neq} E^{0}(\Omega) \stackrel{\complement}{\neq} L^{p}(\Omega)$ for every $p \in\left[1, \infty\left[\right.\right.$. Instead of $H^{2}(\Omega)$ the subspace $H^{2,0}(\Omega)$ of functions for which all generalized second derivatives are in $E^{0}(\Omega)$ is used. In [5] Campanato proves the following regularity result: 

$\left\|\varphi^{*}\right\|_{H^{2,0}} \leq c\|f\|_{L^{2,0}}$ for all $f \in E^{0}(\Omega)$

In [8] Frehse and Rannacher prove the following asymptotic error estimate (for ease $\Omega_{k}=\Omega$ ):

$$
\left\|\varphi_{k}^{*}-\varphi^{*}\right\|_{L^{\infty}} \leq c_{1} h_{k}\left|\ln h_{k}\right| \inf _{\varphi \in \Phi_{k}}\left\|\nabla\left(\varphi-\varphi^{*}\right)\right\|_{L^{\infty}}+c_{2} \inf _{\varphi \in \Phi_{k}}\left\|\varphi-\varphi^{*}\right\|_{L^{\infty}}
$$

Furthermore in [18] Rannacher proves the following (less standard) approximation property of the space $\Phi_{k}$ if $\varphi^{*} \in H^{2,0}(\Omega) \cap H_{0}^{1}(\Omega)$ :

$$
\inf _{\varphi \in \Phi_{k}}\left\{h_{k}\left\|\nabla\left(\varphi-\varphi^{*}\right)\right\|_{L^{\infty}}+\left\|\varphi-\varphi^{*}\right\|_{L^{\infty}}\right\} \leq c h_{k}^{2}\left|\ln h_{k}\right|\left\|\varphi^{*}\right\|_{H^{2,0}}
$$

Combination of (4.5), (4.6) and (4.7) yields (4.4) (note that $\|f\|_{L^{2,0}} \leq c\|f\|_{L^{\infty}}$ ).

TheOREM 4.2. Assume (2.4.a-c) and (3.1.a-e). Then there are constants $k_{0}$ and $C_{A}$ such that for all $k \geq k_{0}$ the following holds:

$$
\left\|L_{k}^{-1}-p L_{k-1}^{-1} r\right\|_{\infty} \leq C_{A} h_{k}^{2}\left|\ln h_{k}\right|^{2}
$$

Proof. Take $g \in U_{k}$. In the proof different constants $C$, all independent of $k$ and $g$, are used. Let $\varphi \in H_{0}^{1}(\Omega), \varphi_{k} \in \Phi_{k}$ and $\varphi_{k-1} \in \Phi_{k-1}$ be such that

$$
\begin{aligned}
& a(\varphi, \psi)=\left(\left(P_{k}^{*}\right)^{-1} g, \psi\right)_{L^{2}} \text { for all } \psi \in H_{0}^{1}(\Omega) \\
& a\left(\varphi_{k}, \psi\right)=\left(\left(P_{k}^{*}\right)^{-1} g, \psi\right)_{L^{2}} \text { for all } \psi \in \Phi_{k} \\
& a\left(\varphi_{k-1}, \psi\right)=\left(\left(P_{k}^{*}\right)^{-1} g, \psi\right)_{L^{2}} \text { for all } \psi \in \Phi_{k-1} .
\end{aligned}
$$

The asymptotic error estimate (4.4) yields that for $k$ large enough, say $k \geq k_{0}$, we have

$$
\left\|\varphi_{m}-\varphi\right\|_{L^{\infty}} \leq C h_{m}^{2}\left|\ln h_{m}\right|^{2}\left\|\left(P_{k}^{*}\right)^{-1} g\right\|_{L^{\infty}} \text { if } m \in\{k, k-1\}
$$

Using (3.1.e) this yields for $k \geq k_{0}$

$$
\left\|\varphi_{k}-\varphi_{k-1}\right\|_{L^{\infty}} \leq C h_{k}^{2}\left|\ln h_{k}\right|^{2}\left\|\left(P_{k}^{*}\right)^{-1} g\right\|_{L^{\infty}}
$$

From (3.7) it follows that $\varphi_{k}=P_{k} L_{k}^{-1} g$ and $\varphi_{k-1}=P_{k-1} L_{k-1}^{-1} r g$. Using Lemma 3.2 and (4.9) we have that for $k \geq k_{0}$ :

$$
\begin{aligned}
& \left\|\left(L_{k}^{-1}-p L_{k-1}^{-1} r\right) g\right\|_{\infty}=\left\|P_{k} L_{k}^{-1} g-P_{k-1} L_{k-1}^{-1} r g\right\|_{L^{\infty}}=\left\|\varphi_{k}-\varphi_{k-1}\right\|_{L^{\infty}} \leq \\
& C h_{k}^{2}\left|\ln h_{k}\right|^{2}\left\|\left(P_{k}^{*}\right)^{-1} g\right\|_{L^{\infty}} \leq C h_{k}^{2}\left|\ln h_{k}\right|^{2}\|g\|_{\infty} .
\end{aligned}
$$




\section{The Smoothing Property}

The usual technique for proving the Smoothing Property requires symmetry (or a nearly symmetric situation), and yields results in the euclidean norm or in the energy norm. We refer to Wittum [24], where smoothing and the construction of smoothers are discussed in a general framework. A new approach to the Smoothing Property has been introduced in [20]. The analysis there does not use symmetry and can also be used for the maximum norm. A disadvantage of this new approach is that we need a damping factor less than or equal to 0.5 (whereas the conditions for the damping factor in [24] are less restrictive). The results of this section can be found in a more general setting in [20]. The analysis here is the same as for the $1 D$ case in [19]. For completeness we give proofs here too.

The smoothing iteration we use is based on a splitting

$$
L_{k}=W_{k}-R_{k}
$$

We make the following assumptions about this splitting:

(5.2.a) $W_{k}$ is regular and $\left\|W_{k}^{-1} R_{k}\right\|_{\infty} \leq 1$ for all $k$,

(5.2.b) $\left\|W_{k}\right\|_{\infty} \leq c h_{k}^{-2}$ for all $k$, with $c$ independent of $k$.

Remark 5.1. It is well-known that if $L_{k}$ is weakly diagonally dominant (i.e. $\sum_{j \neq i}\left|\left(L_{k}\right)_{i j}\right| \leq$ $\left|\left(L_{k}\right)_{i i}\right|$ for all $i$ ) then (5.2.a) holds if (5.1) corresponds to the Jacobi or Gauss-Seidel relaxation. It can also be shown (cf. [21]) that the following criterion holds:

if $L_{k}$ is such that $L_{k} e \geq 0$ (with $e=(1,1, \ldots, 1)^{T}$ and " $\geq$ " entrywise) and if (5.1) is a (weak) regular splitting then (5.2.a) holds.

This criterion applies to the ILU splitting of an $M$-matrix $L_{k}$.

The proof of the Smoothing Property in Theorem 5.3 below is based on the following elementary lemma.

Leмma 5.2. Let $A$ be a square matrix with $\|A\|_{\infty} \leq 1$. Then the following holds

$$
\left\|(I-A)(I+A)^{\nu}\right\|_{\infty} \leq 2\left(\begin{array}{c}
\nu \\
{\left[\frac{1}{2} \nu\right]}
\end{array}\right)<2^{\nu+1} \sqrt{\frac{2}{\pi \nu}} \quad(\nu \geq 1) .
$$

Proof.

$$
(I-A)(I+A)^{\nu}=(I-A) \sum_{k=0}^{\nu}\left(\begin{array}{l}
\nu \\
k
\end{array}\right) A^{k}=I-A^{\nu+1}+\sum_{k=1}^{\nu}\left(\left(\begin{array}{l}
\nu \\
k
\end{array}\right)-\left(\begin{array}{c}
\nu \\
k-1
\end{array}\right)\right) A^{k}
$$

So 


$$
\left\|(I-A)(I+A)^{\nu}\right\|_{\infty} \leq 2+\sum_{k=1}^{\nu}\left|\left(\begin{array}{l}
\nu \\
k
\end{array}\right)-\left(\begin{array}{c}
\nu \\
k-1
\end{array}\right)\right|
$$

Using $\left(\begin{array}{l}\nu \\ k\end{array}\right) \geq\left(\begin{array}{c}\nu \\ k-1\end{array}\right) \Leftrightarrow k \leq \frac{1}{2}(\nu+1)$, and $\left(\begin{array}{c}\nu \\ k\end{array}\right)=\left(\begin{array}{c}\nu \\ \nu-k\end{array}\right)$ we get

$$
\begin{aligned}
& \sum_{k=1}^{\nu}\left|\left(\begin{array}{l}
\nu \\
k
\end{array}\right)-\left(\begin{array}{c}
\nu \\
k-1
\end{array}\right)\right|= \\
& =\sum_{1}^{\left[\frac{1}{2}(\nu+1)\right]}\left(\left(\begin{array}{l}
\nu \\
k
\end{array}\right)-\left(\begin{array}{c}
\nu \\
k-1
\end{array}\right)\right)+\sum_{\left[\frac{1}{2}(\nu+1)\right]+1}^{\nu}\left(\left(\begin{array}{c}
\nu \\
k-1
\end{array}\right)-\left(\begin{array}{l}
\nu \\
k
\end{array}\right)\right) \\
& =\sum_{1}^{\left[\frac{1}{2} \nu\right]}\left(\left(\begin{array}{l}
\nu \\
k
\end{array}\right)-\left(\begin{array}{c}
\nu \\
k-1
\end{array}\right)\right)+\sum_{m=1}^{\left[\frac{1}{2} \nu\right]}\left(\left(\begin{array}{c}
\nu \\
m
\end{array}\right)-\left(\begin{array}{c}
\nu \\
m-1
\end{array}\right)\right) \\
& =2 \sum_{k=1}^{\left[\frac{1}{2} \nu\right]}\left(\left(\begin{array}{l}
\nu \\
k
\end{array}\right)-\left(\begin{array}{c}
\nu \\
k-1
\end{array}\right)\right)=2\left(\left(\begin{array}{c}
\nu \\
{\left[\frac{1}{2} \nu\right]}
\end{array}\right)-\left(\begin{array}{l}
\nu \\
0
\end{array}\right)\right) .
\end{aligned}
$$

Combined with (5.4) this yields the first inequality in (5.3). Elementary analysis yields that

$$
\left(\begin{array}{c}
\nu \\
{\left[\frac{1}{2} \nu\right]}
\end{array}\right)<2^{\nu} \sqrt{\frac{2}{\pi \nu}} \text { for all } \nu \geq 1
$$

For details we refer to [20].

As a smoother we use a damped iteration based on the splitting in (5.1). We consider an iteration with iteration matrix

$$
\left.\left.S_{k}=I-\theta W_{k}^{-1} L_{k}, \quad \theta \in\right] 0, \frac{1}{2}\right]
$$

THEOREM 5.3. Assume that (5.2.a,b) holds. Then we have the following inequality with a constant $C_{S}$ independent of $k$ and $\nu$ :

$$
\left\|L_{k} S_{k}^{\nu}\right\|_{\infty} \leq C_{S} \frac{1}{\sqrt{\nu}} h_{k}^{-2}
$$

Proof. Let $A:=I-2 \theta W_{k}^{-1} L_{k}=2 \theta W_{k}^{-1} R_{k}+(1-2 \theta) I$. Then due to (5.2.a) and $\left.\left.\theta \in\right] 0, \frac{1}{2}\right]$ we have $\|A\|_{\infty} \leq 1$. Using Lemma 5.2 we get

$$
\begin{aligned}
& \left\|L_{k} S_{k}^{\nu}\right\|_{\infty}=\left\|L_{k}\left(I-\theta W_{k}^{-1} L_{k}\right)^{\nu}\right\|_{\infty} \\
& =\left\|\frac{1}{2 \theta} W_{k}(I-A)\left(\frac{1}{2}\right)^{\nu}(I+A)^{\nu}\right\|_{\infty}
\end{aligned}
$$




$$
\begin{aligned}
& \leq \frac{1}{2 \theta}\left\|W_{k}\right\|_{\infty}\left(\frac{1}{2}\right)^{\nu} 2^{\nu+1} \sqrt{\frac{2}{\pi \nu}} \\
& \leq \frac{1}{\theta} \sqrt{\frac{2}{\pi}} c \frac{1}{\sqrt{\nu}} h_{k}^{-2}=: C_{S} \frac{1}{\sqrt{\nu}} h_{k}^{-2} .
\end{aligned}
$$

\section{Contraction number of the two-grid method}

Theorem 4.2 and Theorem 5.3 combined immediately yield the following result.

ThEOREM 6.1. Assume (2.4.a-c), (3.1.a-e) and (5.2.a,b). Let $T_{k}(\nu)$ be as in (3.10) with $S_{k}$ from (5.5). Then there are constants $k_{0}$ and $C_{T G}$ independent of $k$ and $\nu$ such that for all $k \geq k_{0}$, we have

$$
\left\|T_{k}(\nu)\right\|_{\infty} \leq C_{T G} \frac{1}{\sqrt{\nu}}\left|\ln h_{k}\right|^{2}
$$

Clearly Theorem 6.1 shows that if we take $\nu=\nu_{k}$ then for $\left\|T_{k}\left(\nu_{k}\right)\right\|_{\infty} \leq c<1$ to hold, in our upperbound we need $\nu_{k}=g\left(h_{k}\right)$ with $g$ a logarithmically growing function for $h_{k} \downarrow 0$. In $\S 7$ we show that, with the assumptions as in Theorem 6.1 , at least one factor $\left|\ln h_{k}\right|$ is necessary in an upper bound for $\left\|T_{k}(\nu)\right\|_{\infty}$.

With respect to multigrid convergence we note the following. Using the technique as given by Hackbusch in [10] it is straightforward to derive a convergence result in the maximum norm for the multigrid $W$-cycle. A shortcoming of Theorem 6.1 is the requirement $k \geq k_{0}$ (due to the asymptotic error estimates in the proof of the Approximation Property). If we consider a multigrid $W$-cycle with coarsest triangulation $T_{m}$ and if $m<k_{0}$ then we need bounds for $\left\|T_{k}(\nu)\right\|_{\infty}$ with $k<k_{0}$ too. Convergence results in the euclidean norm yield such a bound:

$$
\left\|T_{k}(\nu)\right\|_{\infty} \leq c h_{k}^{-1}\left\|T_{k}(\nu)\right\|_{k} \leq \frac{c}{\nu} h_{k}^{-1}
$$

So we have $\left\|T_{k}(\nu)\right\|_{\infty} \leq c<1$ on coarse grids $\left(k<k_{0}\right)$ if $\nu=\nu_{k} \sim h_{k}^{-1}$. Note that this is not very satisfactory, but also it is of minor importance because it does not affect the order of complexity (for $h_{k} \downarrow 0$ ) of the algorithm.

Remark 6.2. In this paper we only consider the symmetric variational problem (2.1). From $\$ 5$ we see that in the proof of the Smoothing Property (Theorem 5.3) this symmetry is not used; the only conditions are (5.2.a,b). The proof of the Approximation Property (Theorem 4.2) remains valid for a more general (e.g. nonsymmetric) problem, provided that the results in Lemma 3.2 and in (4.4) hold. The results in Lemma 3.2 do not depend on $a(\cdot, \cdot)$ at all, but only on the triangulation. So our analysis yields a result as in Theorem 6.1 for a more general second order elliptic boundary value problem too, if the finite element error estimate (4.4) from $[8,18]$ holds. In $[8,18]$ only the symmetric situation as in $(2.1)$ is considered, however, in [17] it is remarked that (4.4) can be carried over to general second order elliptic problems, 
provided that $\partial \Omega$ and the coefficients are sufficiently regular, and that the corresponding proofs in $[8,18]$ require only very little technical changes.

\section{A lower bound for the contraction number}

In this section we show that the estimate in Theorem 6.1 is sharp in the sense that a factor $\left|\ln h_{k}\right|$ is necessary (the power 2 of the $\left|\ln h_{k}\right|$ term, however, may be due to the method of proof).

We consider the Poisson equation on the unit square and use a linear finite element discretization on a uniform triangulation. We analyze the standard two-grid method as in $\S 3$ for solving the resulting system of equations. We show (cf. Theorem 7.6 and 7.7 below) that for a fixed number of smoothing iterations the maximum norm of the iteration matrix is bounded from below by $C\left|\ln h_{k}\right|$. Our approach is based on the analysis given in [11]. Also we use an important result from [11] (Lemma 7.3 below).

Let $\Omega$ be the unit square and consider the variational boundary value problem as in (2.1), (2.2) with

$$
a(\varphi, \psi):=\int_{\Omega} \nabla \varphi \cdot \nabla \psi d x
$$

We use a uniform triangulation with mesh size parameter $h_{k}=2^{-(k+1)}\left(k \in N_{0}\right)$ as indicated in Fig. 1.

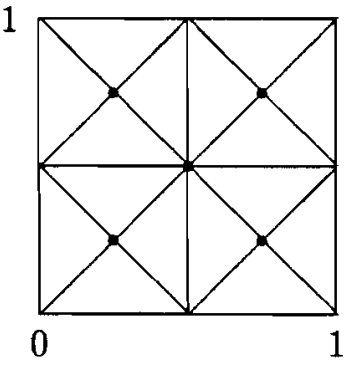

$\mathcal{T}_{0}\left(h_{0}=\frac{1}{2}\right)$

Fig. 1

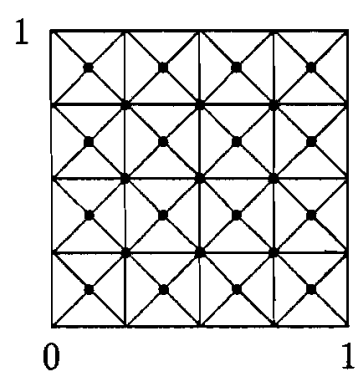

$\mathcal{T}_{1}\left(h_{1}=\frac{1}{4}\right)$

Now let $\mathcal{T}_{k-1}$ and $\mathcal{T}_{k}$ be two successive triangulations. We use the definitions of $\S 3$ and for ease of notation we introduce $h:=h_{k}, \mathcal{T}_{h}:=\mathcal{T}_{k}, J_{h}:=J_{k}, \Phi_{h}:=\Phi_{k}, \varphi_{h}^{i}:=\varphi_{k}^{i}, U_{h}:=U_{k}$, $P_{h}:=P_{k},\left\langle u, v>_{h}:=\langle u, v\rangle_{k}, L_{h}:=L_{k}\right.$. Likewise we use $H:=2 h, \mathcal{T}_{H}, J_{H}, \Phi_{H}, \varphi_{H}^{i}$, $U_{H}, P_{H},<\cdot, \cdot>_{H}, L_{H}$. Furthermore the index in $J_{h}\left(J_{H}\right)$ corresponding to the grid point $Q:=\left(\frac{1}{2}, \frac{1}{2}\right)$ is denoted by $i_{h}^{Q}\left(i_{H}^{Q}\right)$ and the unit vector in $U_{h}\left(U_{H}\right)$ corresponding to $Q$ is denoted by $\boldsymbol{e}_{h}^{Q}\left(e_{H}^{Q}\right)$.

We now introduce a function $\omega_{h} \in \Phi_{h}$ which plays an important role in the analysis below. This function is defined as the piecewise linear interpolant on $\mathcal{T}_{h}$ of the ( $h$-dependent) 


\section{function}

$$
\omega\left(x_{1}, x_{2}\right)=\frac{1}{4} \sin \left(\frac{\pi}{h}\left|x_{1}-\frac{1}{2}\right|\right) \sin \left(\frac{\pi}{h}\left|x_{2}-\frac{1}{2}\right|\right)
$$

We write $w_{h}:=P_{h}^{-1} \omega_{h}$. For all grid points $x_{h}^{i}$ in $\mathcal{T}_{h}$ we have $\omega_{h}\left(x_{h}^{i}\right) \in\left\{-\frac{1}{4}, \frac{1}{4}, 0\right\}$. In Fig. 2 we show the pattern of these function values.

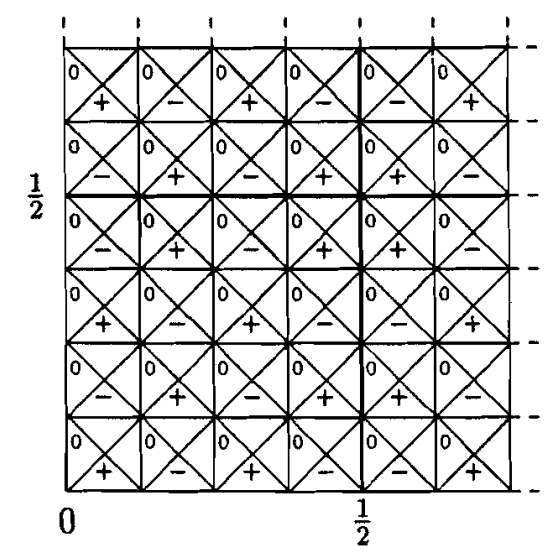

Fig. 2 Values of the function $\omega_{h}$. + : value $\frac{1}{4} ;-:$ value $-\frac{1}{4} ; 0$ : value 0 .

It is easy to prove Lemma 7.1 below. A simple proof can be found e.g. in [11].

LEMMA 7.1. For $i, j \in J_{h}$ the following holds

$$
a\left(\varphi_{h}^{i}, \varphi_{h}^{j}\right)=\left\{\begin{aligned}
4 & \text { if } i=j \\
-1 & \text { if }\left|x_{h}^{i}-x_{h}^{j}\right|=\frac{1}{2} \sqrt{2} h \\
0 & \text { otherwise. }
\end{aligned}\right.
$$

LEMMA 7.2. The following holds:

$$
L_{h} w_{h}=h^{-2}\left(4 w_{h}-e_{h}^{Q}\right)
$$

and for $i \in J_{H}$ :

$$
a\left(\omega_{h}, \varphi_{H}^{i}\right)=\left\{\begin{array}{lll}
0 & \text { if } \quad i \neq i_{H}^{Q} \\
1 & \text { if } \quad i=i_{H}^{Q} .
\end{array}\right.
$$


Proof. We extend the vector $w_{h} \in U_{h}$ by taking zero values corresponding to all boundary grid points of $\mathcal{T}_{h}$. Then Lemma 7.1 yields that $h^{2} L_{h}$ corresponds to the difference star

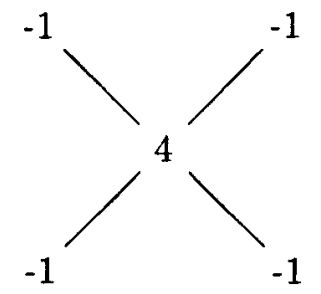

in all interior points.

For $i \in J_{h}$ the value $\left(h^{2} L_{h} w_{h}\right)_{i}$ can be found by application of this difference star in the grid point $x_{h}^{i}$ to the function $\omega_{h}=P_{h} w_{h}$. Fig. 2 then shows that

$$
\left(h^{2} L_{h} w_{h}\right)_{i}= \begin{cases}4\left(w_{h}\right)_{i} & \text { if } i \neq i_{h}^{Q} \\ -1 & \text { if } i=i_{h}^{Q}\end{cases}
$$

So $h^{2} L_{h} w_{h}=4 w_{h}-e_{h}^{Q}$ holds.

The proof of (7.4) runs as follows. For $i \in J_{H}$ we have

$$
a\left(\omega_{h}, \varphi_{H}^{i}\right)=<L_{h} w_{h}, P_{h}^{-1} \varphi_{H}^{i}>_{h}=4 h^{-2}<w_{h}, P_{h}^{-1} \varphi_{H}^{i}>_{h}-h^{-2}<e_{h}^{Q}, P_{h}^{-1} \varphi_{H}^{i}>_{h} .
$$

Note that

$$
h^{-2}<e_{h}^{Q}, P_{h}^{-1} \varphi_{H}^{i}>_{h}=\left(P_{h}^{-1} \varphi_{H}^{i}\right)_{i_{h}^{Q}}=\left\{\begin{array}{cc}
0 & \text { if } i \neq i_{H}^{Q} \\
1 & \text { if } i=i_{H}^{Q}
\end{array}\right.
$$

With respect to the term $4 h^{-2}<w_{h}, P_{h}^{-1} \varphi_{H}^{i}>_{h}$ in (7.5) we note the following. First we take $i \in J_{H}$ such that $x_{H}^{i}$ is a vertex of 4 triangles in $\mathcal{T}_{H}$. Then $\varphi_{H}^{i}$ has nonzero values in 5 grid points of $\mathcal{T}_{h}$ as indicated in Fig. 3. Using Fig. 2 we see that in these grid points $\omega_{h}$ has values as indicated in Fig. 3.

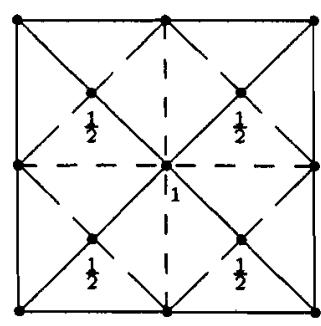

$\varphi_{H}^{i}$

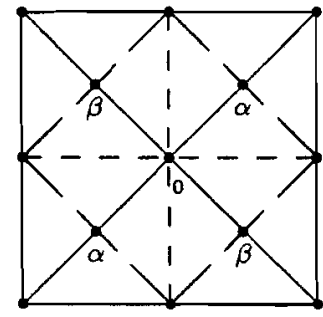

$\alpha, \beta \in\left\{-\frac{1}{4}, \frac{1}{4}\right\}$ $\alpha+\beta=0$

- : triangles in $\mathcal{T}_{H}$

So $4 h^{-2}<w_{h}, P_{h}^{-1} \varphi_{H}^{i}>_{h}=0$. Secondly, for $i \in J_{H} \backslash\left\{i_{H}^{Q}\right\}$ such that $x_{H}^{i}$ is a vertex of 8 triangles in $T_{H}$ we have values for $\varphi_{H}^{i}$ and $\omega_{h}$ as illustrated in Fig. 4. 


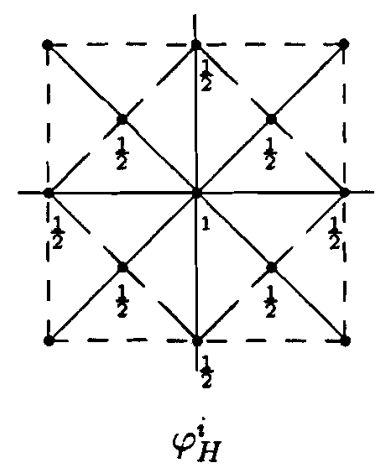

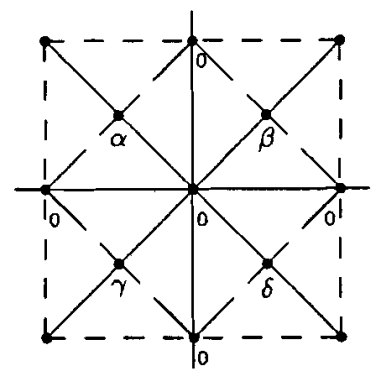

$\omega_{h}$ $\alpha, \beta, \gamma, \delta \in\left\{-\frac{1}{4}, \frac{1}{4}\right\}$
$\alpha+\beta+\gamma+\delta=0$
-: triangles in $\mathcal{T}_{H}$

Fig. 4

So again $4 h^{-2}<w_{h}, P_{h}^{-1} \varphi_{H}^{i}>_{h}=0$. Finally for $i=i_{H}^{Q}$ we have a situation as in Fig. 4 but now with $\alpha=\beta=\gamma=\delta=\frac{1}{4}$, so $4 h^{-2}<w_{h}, P_{h}^{-1} \varphi_{H}^{i}>_{h}=2$.

Using these results for $4 h^{-2}<w_{h}, P_{h}^{-1} \varphi_{H}^{i}>_{h}$ and the result (7.6) in (7.5) proves (7.4).

A proof of the following lemma is given by Haverkamp in [11; Lemma 5].

LEMMA 7.3. If $\psi_{H} \in \Phi_{H}$ is such that for $i \in J_{H}$ we have

$$
a\left(\psi_{H}, \varphi_{H}^{i}\right)= \begin{cases}0 & \text { if } i \neq i_{H}^{Q} \\ 1 & \text { if } i=i_{H}^{Q}\end{cases}
$$

then $\psi_{H}(Q)>\pi^{-2}|\ln H|$.

LEMMA 7.4. The following holds:

$$
\left\|\left(I-p L_{H}^{-1} r L_{h}\right) w_{h}\right\|_{\infty}>\pi^{-2}|\ln (2 h)|
$$

Proof. We take $\psi_{H}$ as in Lemma 7.3. Then it follows (cf. Lemma 7.2) that $\psi_{H}$ is the orthogonal projection of $\omega_{h}$ on $\Phi_{H}$ :

$$
a\left(\psi_{H}, \varphi_{H}^{i}\right)=a\left(\omega_{h}, \varphi_{H}^{i}\right) \text { for all } i \in J_{H}
$$

So

$$
<L_{H} P_{H}^{-1} \psi_{H}, P_{H}^{-1} \varphi_{H}^{i}>_{H}=<r L_{h} w_{h}, P_{H}^{-1} \varphi_{H}^{i}>_{H} \text { for all } i \in J_{H} \text {, }
$$

and thus

$$
\psi_{H}=P_{H} L_{H}^{-1} r L_{h} w_{h}
$$

This yields 


$$
\begin{aligned}
& \left\|\left(I-p L_{H}^{-1} r L_{h}\right) w_{h}\right\|_{\infty}=\left\|P_{h}\left(I-p L_{H}^{-1} r L_{h}\right) w_{h}\right\|_{\infty} \\
& =\left\|\omega_{h}-\psi_{H}\right\|_{\infty} \geq\left|\left(\omega_{h}-\psi_{H}\right)(Q)\right|=\left|\psi_{H}(Q)\right|>\pi^{-2}|\ln (2 h)| .
\end{aligned}
$$

We now prove three theorems in which the main results of this section are given. Theorem 7.5 gives an estimate related to the Approximation Property in Theorem 4.2. In Theorem 7.6 and Theorem 7.7 we give lower bounds for $\left\|T_{k}(\nu)\right\|_{\infty}$. In Theorem 7.6 we take damped Jacobi and damped Gauss-Seidel as a smoother with a damping parameter $\theta \in] 0, \frac{1}{2}[$. In Theorem 7.7 we consider damped Jacobi again, but then with $\theta \in] 0,1[$.

THEOREM 7.5. The following holds:

$$
\left\|L_{h}^{-1}-p L_{H}^{-1} r\right\|_{\infty}>\pi^{-2} h^{2}|\ln (2 h)| \text {. }
$$

Proof. From Lemma 7.2 we have $\left\|L_{h} w_{h}\right\|_{\infty}=h^{-2}\left\|4 w_{h}-e_{h}^{Q}\right\|_{\infty}=h^{-2}$. Lemma 7.4 then yields

$$
\left\|L_{h}^{-1}-p L_{H}^{-1} r\right\|_{\infty} \geq\left\|\left(L_{h}^{-1}-p L_{H}^{-1} r\right) L_{h} w_{h}\right\|_{\infty} /\left\|L_{h} w_{h}\right\|_{\infty}>\pi^{-2} h^{2}|\ln (2 h)|
$$

We consider a splitting as in (5.1) corresponding to the Jacobi or Gauss-Seidel method. Note that the conditions $(5.2 . \mathrm{a}, \mathrm{b})$ are fulfilled. We use a damped version with damping parameter $\theta\left(S_{h}=I-\theta W_{h}^{-1} L_{h}\right)$.

THEOREM 7.6. For $\theta \in] 0, \frac{1}{2}[$ the following holds:

$$
\left\|T_{h}(\nu)\right\|_{\infty}>4 \pi^{-2}(1-2 \theta)^{\nu}|\ln (2 h)| \quad(\nu \geq 0) .
$$

Proof. $\left\|I-W_{h}^{-1} L_{h}\right\|_{\infty} \leq 1$ holds and thus $\left\|W_{h}^{-1} L_{h}\right\|_{\infty} \leq 2$. So for $\left.\theta \in\right] 0, \frac{1}{2}[$ we have

$$
\left\|\left(I-\theta W_{h}^{-1} L_{h}\right)^{-1}\right\|_{\infty} \leq\left(1-\theta\left\|W_{h}^{-1} L_{h}\right\|_{\infty}\right)^{-1} \leq(1-2 \theta)^{-1}
$$

Combining (7.7), Lemma 7.4 and $\left\|w_{h}\right\|_{\infty}=\frac{1}{4}$ yields

$$
\begin{aligned}
& \left\|T_{h}(\nu)\right\|_{\infty}=\left\|\left(I-p L_{H}^{-1} r L_{h}\right) S_{h}^{\nu}\right\|_{\infty} \\
& \geq\left\|\left(I-p L_{H}^{-1} r L_{h}\right) w_{h}\right\|_{\infty} /\left\|S_{h}^{-\nu} w_{h}\right\|_{\infty}>\pi^{-2}|\ln (2 h)|(1-2 \theta)^{\nu} 4 .
\end{aligned}
$$

THEOREM 7.7. Let $S_{h}=I-\theta \frac{1}{4} h^{2} L_{h}$ be the iteration matrix corresponding to the damped Jacobi iteration. Then for $\theta \in] 0,1[$ the following holds with a constant $K$ independent of $h$ :

$$
\left\|T_{h}(\nu)\right\|_{\infty}>4 \pi^{-2}(1-\theta)^{\nu}|\ln (2 h)|-K \quad(\nu \geq 0) .
$$


Proof. The following holds (cf. Lemma 7.2):

$$
S_{h} w_{h}=\left(I-\theta \frac{1}{4} h^{2} L_{h}\right) w_{h}=(1-\theta) w_{h}+\frac{1}{4} \theta e_{h}^{Q}
$$

Thus

$$
S_{h}^{\nu} w_{h}=(1-\theta)^{\nu} w_{h}+\frac{1}{4} \theta \sum_{k=0}^{\nu-1}(1-\theta)^{k} S_{h}^{\nu-1-k} e_{h}^{Q}
$$

The analysis of the coarse grid correction w.r.t. the scaled euclidean norm $\|\cdot\|_{h}=\langle\cdot, \cdot\rangle_{h}^{\frac{1}{2}}$ yields (see e.g. [10]):

$$
\left\|I-p L_{H}^{-1} r L_{h}\right\|_{h} \leq K, \quad K \text { independent of } h
$$

Also note that for every $\theta \in] 0,1[$ we have

$$
\left\|S_{h}^{m} e_{h}^{Q}\right\|_{h} \leq\left\|S_{h}^{m}\right\|_{h}\left\|e_{h}^{Q}\right\|_{h} \leq h \quad(m \geq 0)
$$

Combination of Lemma 7.4, (7.8), (7.9), (7.10) and $\|u\|_{\infty} \leq h^{-1}\|u\|_{h}$ yields

$$
\begin{aligned}
& \left\|\left(I-p L_{h}^{-1} r L_{h}\right) S_{h}^{\nu}\right\|_{\infty} \geq 4\left\|\left(I-p L_{H}^{-1} r L_{h}\right) S_{h}^{\nu} w_{h}\right\|_{\infty} \\
& \quad \geq 4(1-\theta)^{\nu}\left\|\left(I-p L_{H}^{-1} r L_{h}\right) w_{h}\right\|_{\infty}-\theta\left\|\left(I-p L_{H}^{-1} r L_{h}\right) \sum_{k=0}^{\nu-1}(1-\theta)^{k} S_{h}^{\nu-1-k} e_{h}^{Q}\right\|_{\infty} \\
& \quad>4(1-\theta)^{\nu} \pi^{-2}|\ln (2 h)|-\theta h^{-1} K \sum_{k=0}^{\nu-1}(1-\theta)^{k} h \\
& >4(1-\theta)^{\nu} \pi^{-2}|\ln (2 h)|-K .
\end{aligned}
$$

Acknowledgement. The author would like to thank Prof.dr. R. Rannacher for some helpful comments.

\section{References}

[1] R.E. BANK AND C.C. Douglas, Sharp estimates for multigrid rates of convergence with general smoothing and acceleration, SIAM J. Numer. Anal., 22 (1985), pp. 617633.

[2] D. BRAESS, The contraction number of a multigrid method for solving the Poisson equation, Numer. Math., 37 (1981), pp. 387-404. 
[3] J.H. Bramble and J.E. Pasciak, New convergence estimates for multigrid algorithms, Math. Comp., 49 (1987), pp. 311-329.

[4] J.H. Bramble, J.E. Pasciak, J. Wang, J. Xu, Convergence estimates for multigrid algorithms without regularity assumptions, Math. Comp., 57 (1991), pp. 23-45.

[5] S. Campanato, Equazioni ellitiche del IP ordine e spazi $\mathcal{L}^{(2, \lambda)}$, Ann. Mat. Pura Appl., 69 (1965), pp. 321-381.

[6] N.H. Decker, J. MANDEL, S.V. PaRTer, On the role of regularity in multigrid methods, in Multigrid Methods: theory, application and supercomputing, Lecture Notes in Pure and Applied Mathematics 110, Marcel Dekker Inc., 1988.

[7] J. Descloux, On finite element matrices, SIAM J. Numer. Anal., 9 (1972), pp. 260-265.

[8] J. FREHSE AND R. RANNACHER, Eine $L^{1}$-Fehlerabschätzung für diskrete Grundlösungen in der Methode der finiten elemente, Tagungsband "Finite Elemente" Bonn. Math. Schr. 1976.

[9] P. Grisvard, Elliptic problems in nonsmooth domains, Pitman, Boston-LondonMelbourne, 1985.

[10] W. Hасквusch, Multi-grid methods and applications, Springer, Berlin, 1985.

[11] R. Haverkamp, Eine Aussage zur $L_{\infty}$-Stabilität und zur genauen Konvergenzordnung der $H_{0}^{1}$-Projektionen, Numer. Math., 44 (1984), pp. 393-405.

[12] F. John AND L. NIRENBERg, On functions of bounded mean oscillation, Comm. Pure Appl. Math., 14 (1961), pp. 415-426.

[13] J. MANDEL, S. MCCormick, R. BANK, Variational multigrid theory, in [14], pp. $131-178$.

[14] S. MCCormick (ED.), Multigrid Methods, SIAM Frontiers in Applied Math. 5, SIAM, Philadelphia, 1987.

[15] J. NITSCHE, $L_{\infty}$-convergence of finite element approximations, in Mathematical aspects of finite element methods, Lecture Notes in Math. 606, pp. 261-274.

[16] S.V. Parter, Remarks on multigrid convergence theorems, Appl. Math. Comp., 23 (1987), pp. 103-120.

[17] R. RANNACHER, private communication (1991).

[18] R. RANNACHER, Zur $L^{\infty}$-Konvergenz linearer finiter elemente beim Dirichlet-problem, Math. Z., 149 (1976), pp. 69-77.

[19] A. REUSKEN, On maximum norm convergence of multigrid methods for two-point boundary value problems, to appear in SIAM J. Numer. Anal.

[20] A. Reusken, A new lemma in multigrid convergence theory, RANA report 91-07, Department of Mathematics and Computing Science, Eindhoven University of Technology, 1991. 
[21] A. Reusken, The Smoothing Property for regular splittings, submitted for Proceedings of the Eight GAMM-Seminar Kiel on Incomplete Decompositions, 1992.

[22] A.H. Schatz And L.B. Wahlbin, Maximum norm estimates in the finite element method on plane polygonal domains. Part I, Math. Comp., 32 (1978), pp. 73-109.

[23] R. Scotr, Optimal $L^{\infty}$ estimates for the finite element method on irregular meshes, Math. Comp., 30 (1976), pp. 681-697.

[24] G. Wittum, Linear iterations as smoothers in multigrid methods: theory with applications to incomplete decompositions, Impact of Computing in Science and Engineering, 1 (1989), pp. 180-215. 